\title{
Assessment of lumbar paraspinal muscle activation using fMRI BOLD imaging and T2 mapping
}

\author{
Yi-Long Huang ${ }^{1 \#}$, Jia-Long Zhou ${ }^{1,2 \#}$, Yuan-Ming Jiang ${ }^{1}$, Zhen-Guang Zhang ${ }^{1}$, Wei Zhao ${ }^{1}$, Dan Han ${ }^{1}$, \\ Bo $\mathrm{He}^{1}$
}

${ }^{1}$ Department of Medical Imaging, The First Affiliated Hospital of Kunming Medical University Yunnan, Kunming 650032, China; ${ }^{2}$ Department of Magnetic Resonance Imaging, The First People's Hospital of Yunnan Province, Kunming 650032, China

"These authors contributed equally to this work.

Correspondence to: Dr. Bo He, MD. Department of Medical Imaging, The First Affiliated Hospital of Kunming Medical University Yunnan, No. 295 Xichang Road, Kunming 650032, China. Email: 929883137@qq.com; Dr. Dan Han, MD. Department of Medical Imaging, The First Affiliated Hospital of Kunming Medical University Yunnan, No. 295 Xichang Road, Kunming 650032, China. Email: kmhandan@sina.com.

\begin{abstract}
Background: Our study aimed to investigate the feasibility of functional magnetic resonance imaging [blood oxygen level-dependent (BOLD) imaging and T2 mapping] in monitoring the activation of lumbar paraspinal muscles before and after exercise.

Methods: The ethics committee of the First Affiliated Hospital of Kunming Medical University approved our study. Both BOLD and T2 mapping of paraspinal muscles were performed in 50 healthy, young volunteers before and after upper-body extension exercises. The movement tasks included upper body flexion and extension using a simple Roman chair. Cross-sectional area (CSA), R2*, and T2 values were measured in various lower-back anatomical regions. The SPSS22.0 statistical software was used to analyze all the data.

Results: Post-exercise CSA and T2 values were higher than those recorded in the pre-exercise session for the three lower-back muscles that were evaluated (iliocostalis, longissimus, and multifidus) $(\mathrm{P}<0.01)$. However, R2* values of these muscles were significantly lower after exercise $(\mathrm{P}<0.01)$. A significant difference in the R2*, CSA, and T2 values of the iliocostalis occurred between males and females $(\mathrm{P}<0.05)$. No statistically significant differences were evident for R2*, CSA, and T2 of the lower-back muscles between L3 and L4 levels, or between the left and right sides. The total CSA of the iliocostalis was higher than that of the multifidus and longissimus $(\mathrm{P}<0.05)$.
\end{abstract}

Conclusions: BOLD and T2 mapping are feasible non-invasive indirect assessments of lumbar paraspinal muscle activation before and after exercise.

Keywords: Blood oxygen level-dependent (BOLD); cross-sectional area (CSA); functional magnetic resonance imaging (fMRI); muscle activation; paraspinal muscle; T2 mapping

Submitted Jun 21, 2019. Accepted for publication Oct 22, 2019.

doi: 10.21037 /qims.2019.10.20

View this article at: http://dx.doi.org/10.21037/qims.2019.10.20

\section{Introduction}

Lumbar paraspinal muscles are involved in motor function and stability of the lower back. Magnetic resonance imaging (MRI) or computed tomography-based cross-sectional area (CSA) of the lumbar paraspinal muscles shows muscle activation and has been used to assess muscular recovery during rehabilitation training in patients with low back pain (1-4). However, it has been reported that CSA is not sensitive in detecting atrophy of the lumbar paraspinal muscles $(4,5)$. The atrophied paraspinal muscle tissue may be replaced by fat and fibrous tissue, resulting in insignificant 
Table 1 Baseline characteristics of the 50 volunteers (mean \pm standard deviation)

\begin{tabular}{lcccc}
\hline Sex & Age (year) & Height $(\mathrm{cm})$ & Weight $(\mathrm{kg})$ & $\mathrm{BMI}\left(\mathrm{kg} / \mathrm{m}^{2}\right)$ \\
\hline $\begin{array}{l}\text { Female } \\
(\mathrm{n}=25)\end{array}$ & $24.73 \pm 2.06$ & $159.15 \pm 4.96$ & $52.27 \pm 6.78$ & $20.59 \pm 1.95$ \\
$\begin{array}{l}\text { Male } \\
(\mathrm{n}=25)\end{array}$ & $26.09 \pm 2.84$ & $169.33 \pm 3.23$ & $63.71 \pm 5.46$ & $22.22 \pm 1.75$ \\
$P$ value & 0.121 & $<0.001$ & $<0.001$ & 0.073 \\
\hline
\end{tabular}

BMI, body mass index.

changes in total CSA. However, this may lead to reduced muscle activation, which can affect microcirculatory blood perfusion, water metabolism, and energy metabolism. Notably, variations in CSA only detect morphological changes of the paraspinal muscles. However, muscular changes may involve both the composition and function of the muscles (6). Thus, monitoring changes in the total CSA of paraspinal muscles is not a suitable marker to evaluate muscle activation.

Blood oxygen level-dependent (BOLD) MRI is a commonly used technique to assess neuronal activation $(7,8)$. Since BOLD is based on the principle that the transverse relaxation rate $\mathrm{R} 2 *\left(1 / \mathrm{T} 2^{*}\right)$ depends on the ratio of deoxyhemoglobin to oxyhemoglobin in the blood surrounding muscle tissue (7-10), it may be used to assess the peripheral microcirculation in skeletal muscle tissue. In contrast, T2 mapping measures tissue transverse relaxation time, which can be used as a quantitative index of changes in tissue composition, water metabolism, lactic acid metabolism, fat degeneration, and other biochemical changes (11). Muscle BOLD and T2 mapping have been used to detect disturbances in microcirculatory blood perfusion and biochemical metabolism in some diseases such as peripheral arterial occlusive disease, Duchenne muscular dystrophy, cartilage diseases, and ischemic heart disease (12-15). Whether BOLD and T2 mapping is superior to CSA for evaluating muscle activation after exercise has not been investigated.

We speculate that functional MRI (fMRI) using BOLD and T2 mapping is feasible to evaluate muscle activation in addition to CSA. The purpose of our study was to compare the fMRI parameters (CSA, R2*, and T2 values) of three paraspinal muscles (iliocostalis, longissimus, and multifidus) between pre-exercise and post-exercise sessions in young, healthy volunteers.

\section{Methods}

\section{Selection of volunteers}

Our study was approved by the ethics committee of our hospital. A total of 50 young, healthy volunteers (25 males and 25 females) were recruited from the radiology department of our affiliated hospital (the First Affiliated Hospital of Kunming Medical University). All participants gave written informed consent before the study. The average age of the volunteers was $24.81 \pm 2.29$ years, with an age range of 19-29 years. The clinical baseline characteristics of the study population are shown in Table 1 .

All 50 volunteers displayed negative MR lumbar examinations. No participants had low back pain or lumbar disk herniation. Other exclusion criteria included a history of lumbar surgery, scoliosis, visible or radiological muscle atrophy, professional paraspinal muscle training, and MRI contraindications such as pacemakers, ferromagnetic implants, and claustrophobia.

\section{Study design and lower-back exercise protocols}

All 50 volunteers who were examined using MRI were allowed $30 \mathrm{~min}$ of rest before starting the exercise. To exclude participants with pathological findings such as lumbar disc herniation, general lumbar sagittal T2weighted imaging, and lumbar disc axial T1-weighted imaging were performed before commencing the exercise. Following initial BOLD and T2 mapping in the resting state, lower-back exercises were performed for $10 \mathrm{~min}$ outside the scanner room. Lower-back hyperextensions using a 45-degree Roman chair for support were performed. This is an isotonic upper-body extension exercise that works the lower back, specifically the erector spinae muscles. Volunteers could adjust the upper Roman chair pad height, leaving enough room to bend at the waist without restriction. The steps included in the exercises were as follows: (I) hands were crossed behind the head, and the body was kept straight; (II) the subject began to slowly bend forward at the waist as far as possible, while keeping the back flat; (III) the subject slowly raised his/her torso back to the original position (Figure 1). Participants were requested to repeat the combined movements 15 times as one set of actions. The entire task consisted of three sets with intervals of $30 \mathrm{~s}$. After completing all actions, BOLD and T2 mapping were repeated using the scanning protocols during 


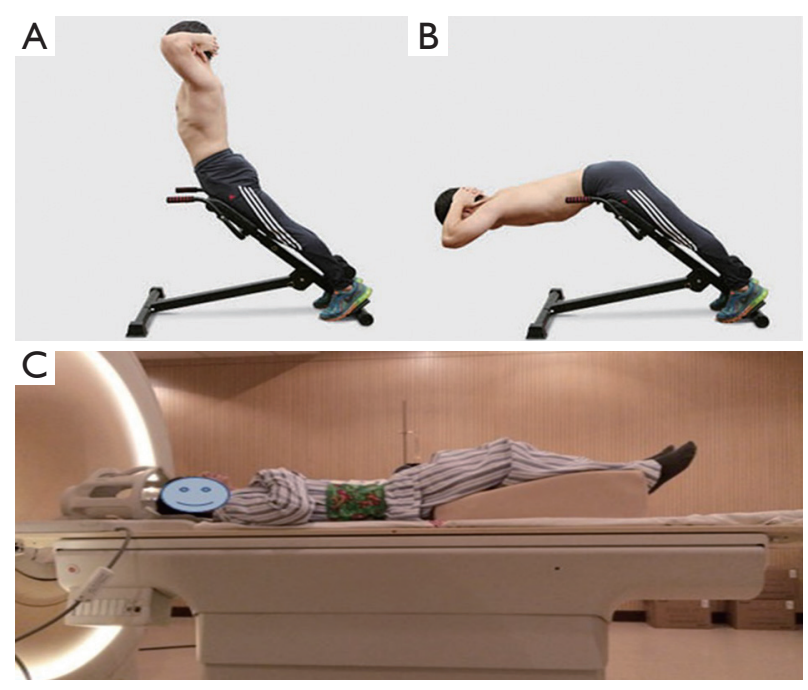

Figure 1 The posture of volunteers and MRI examination position. The volunteer performed lower back hyperextension using a 45-degree Roman chair for support (A and B); (C) The volunteer was in a standard supine position with a big wedgeshaped foam pad under the lower limbs. The hands were placed on the chest.

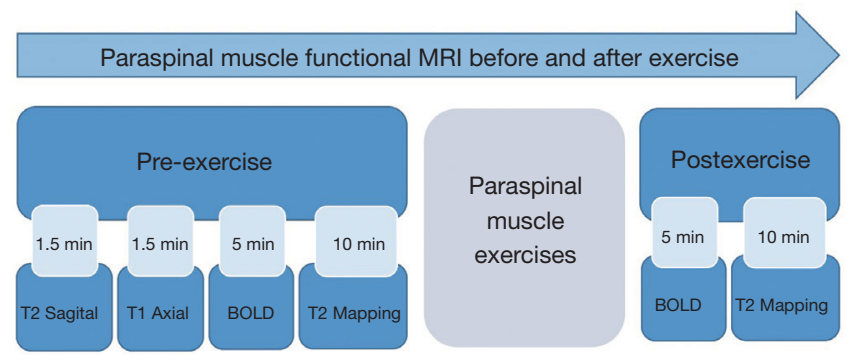

Figure 2 MRI examination protocol for paraspinal muscle function. Duration of pre-exercise (18 min), exercise (10 min), and post-exercise (15 min). BOLD and T2 mapping imaging were collected before and after the exercise ( 5 and $10 \mathrm{~min}$, respectively).

the rest state (Figure 2).

\section{MRI}

The MRI scans were performed on a 3.0T whole-body MR scanner (Achieva 3.0T Tx, Philips Healthcare, Best, Netherlands) using a dedicated sensitivity encoding body coil (Philips Healthcare). Volunteers were requested to compress the abdomen with an abdominal bandage during MR examination to reduce respiratory motion artifacts.
Volunteers were placed in a standard supine position, and a wedge-shaped foam pad was placed under the lower limbs. Hands were placed on the chest to avoid potential aliasing artifacts (Figure 1C).

The MRI scans of the lower back were performed twice for each volunteer. The central lines of the pre- and postexercise scans were placed at the superior margin of the L4 vertebral body and were parallel to each other. The spine was fixed, and the anatomical landmarks were also fixed. This ensured that the two scans were in the same space, and the CSA, $2^{*}$, and $\mathrm{T} 2$ values were all in the same slice. The first examination protocol involved sagittal T2-weighted images of lumbar spines (Figure $3 A, B$ ), axial T1-weighted images of intervertebral discs, and muscle BOLD and T2 mapping. The BOLD multi-echo fast field echo (mFFE) and T2 mapping images were acquired in the transverse orientation, and slice positions were aligned perpendicularly to the superior margins of the L3-L4 levels of volunteers using sagittal T2-weighted images. The muscle BOLD acquisition parameters were as follows: field of view $($ FOV $)=100 \times 218 \times 72 \mathrm{~mm}^{3}$; repetition time $(\mathrm{TR})=466 \mathrm{~ms}$; number of signals averaged $=6$; voxel $=1.5 \times 1.5 \times 3.0 \mathrm{~mm}^{3}$; slice thickness $=3.0 \mathrm{~mm}$; and slice increment $=0.0 \mathrm{~mm}$. Six echo images with nearly identical increasing echo times (TE) were acquired: $\mathrm{TE}=3.7,10.3,15.9,21.5,27.1$, and $32.7 \mathrm{~ms}$; and the fat-suppression technique was applied to BOLD scanning. The T2 mapping was based on a fast spinecho sequence without fat-suppression: $F O V=100 \times 211 \times$ $79 \mathrm{~mm}^{3}$; TR $=1,974 \mathrm{~ms}$; number of signal averaged $=1$; voxel $=0.76 \times 0.76 \times 3.0 \mathrm{~mm}^{3}$; slice thickness $=3.0 \mathrm{~mm}$; slice increment $=0.7 \mathrm{~mm}$; and $\mathrm{TE}=7,13,19,25,31$, and $37 \mathrm{~ms}$ (Table 2).

\section{Data measurement and analysis}

Results were obtained before and after exercise in duplicate for both BOLD and T2 mapping. Image analysis was performed using a commercial workstation (Advantage Windows 4.4, GE Medical Systems, USA). The images were obtained by two radiologists with over 5 years of experience in MRI and musculoskeletal system physiology. The radiologists were blinded to the study design. Muscle CSA, R2*, and T2 values of the bilateral lower-back muscles were obtained on a region of interest (ROI) basis at the superior margins of the L 3 and L4 vertebral bodies. The radiologists manually delineated the shape of the bilateral iliocostalis muscles, longissimus muscles, and multifidus muscles on the superior margins of the L3 and L4 vertebral 

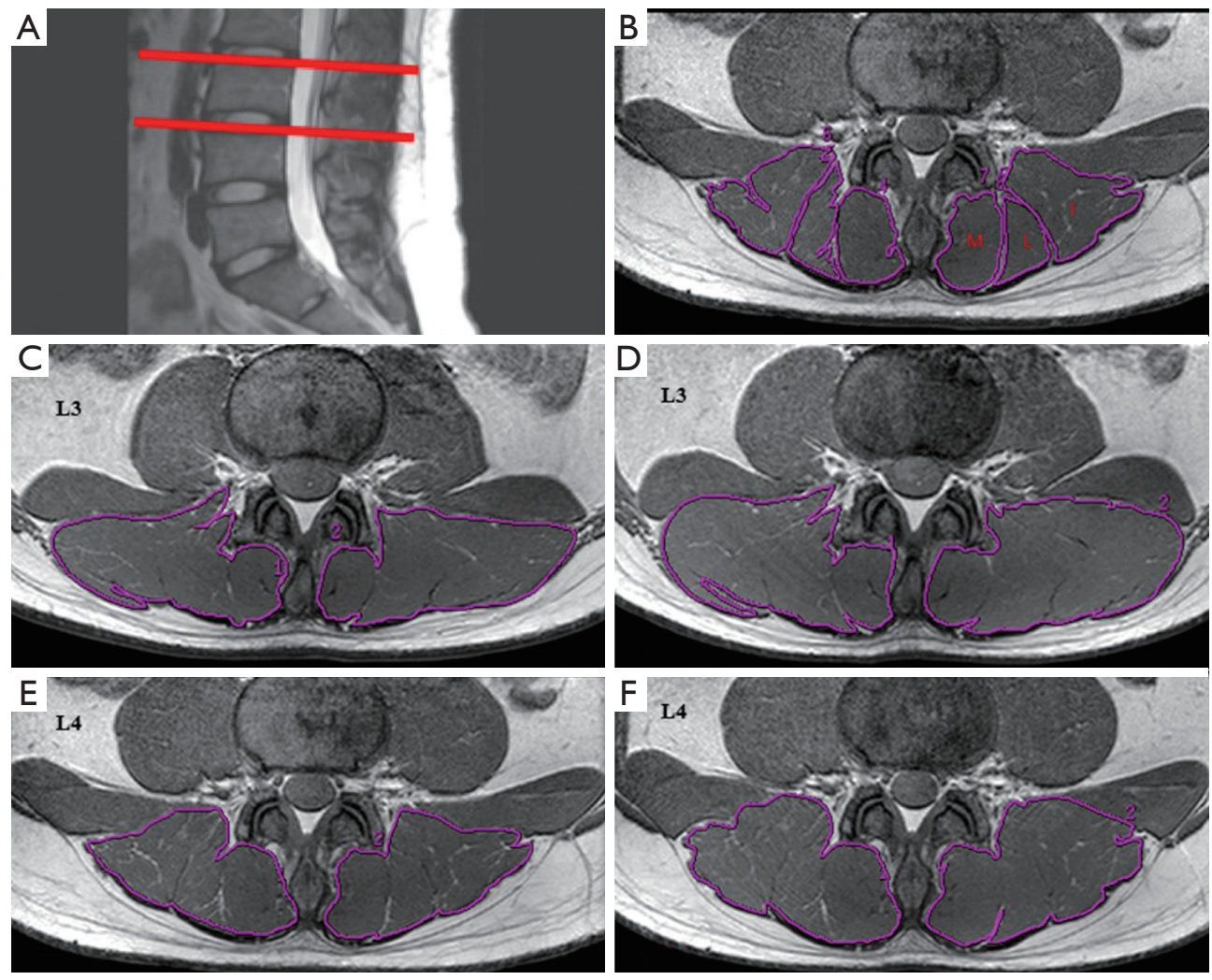

Figure 3 MRI images. (A) The superior margins of the L3 and L4 were the regions of interests. (B) Muscle identity: I, iliocostalis; L, longissimus; and M, multifidus. (C,D) T2 mapping raw images obtained before exercise at L3. (E,F) T2 mapping raw images obtained before exercise at L4.

Table 2 MRI scan parameters

\begin{tabular}{|c|c|c|c|c|c|c|c|c|}
\hline Images & $\begin{array}{c}\text { Pulse } \\
\text { sequence }\end{array}$ & Voxel & TE (ms) & $\mathrm{TR}(\mathrm{ms})$ & $\begin{array}{c}\text { Number of signal } \\
\text { averaged }\end{array}$ & $\mathrm{ST}(\mathrm{mm})$ & $\mathrm{SL}(\mathrm{mm})$ & $\begin{array}{c}\text { Slice } \\
\text { number }\end{array}$ \\
\hline $\mathrm{T} 2 \mathrm{WI}$ & FSE & $0.91 \times 1.32 \times 4.00$ & 120 & 2,000 & 2 & 4 & 0.4 & 9 \\
\hline BOLD & mFEE & $1.50 \times 1.50 \times 4.00$ & $3.7 / 10.3 / 15.9 / 21.5 / 27.1 / 32.7$ & 466 & 6 & 3 & 0 & 24 \\
\hline T2 mapping & FSE & $0.76 \times 0.76 \times 0.30$ & $7 / 13 / 19 / 25 / 31 / 37$ & 1,974 & 1 & 3 & 0.7 & 24 \\
\hline
\end{tabular}

BOLD, blood oxygen level dependent; mFFE, multi-echoes fast field echo; MRI, magnetic resonance imaging; FSE, fast spin echo; SL, slice increment; ST, slice thickness; T1WI, T1-weighted imaging; T2WI, T2-weighted imaging; TE, echo time.

bodies (Figure 3B). The ROIs of the CSA were drawn on the T2 mapping raw images (Figure $3 C, D, E, F$ ) and copied ontoT2 maps from which T2 values were obtained. The ROIs of the R2* maps were drawn separately, and each ROI was measured three times. The average of the three measurements was calculated and used for later analysis. The tendons and muscle fat were avoided during the measurement process.

\section{Statistical analysis}

All analyses were performed using the statistical software SPSS for Windows version 22.0 (IBM SPSS Inc., IL, USA). A $\mathrm{P}$ value $<0.05$ was considered statistically significant. All measured values were expressed as mean \pm standard deviation. A paired student $t$-test was used to compare differences in the CSA, $\mathrm{R} 2{ }^{*}$, and $\mathrm{T} 2$ of lumbar paraspinal 

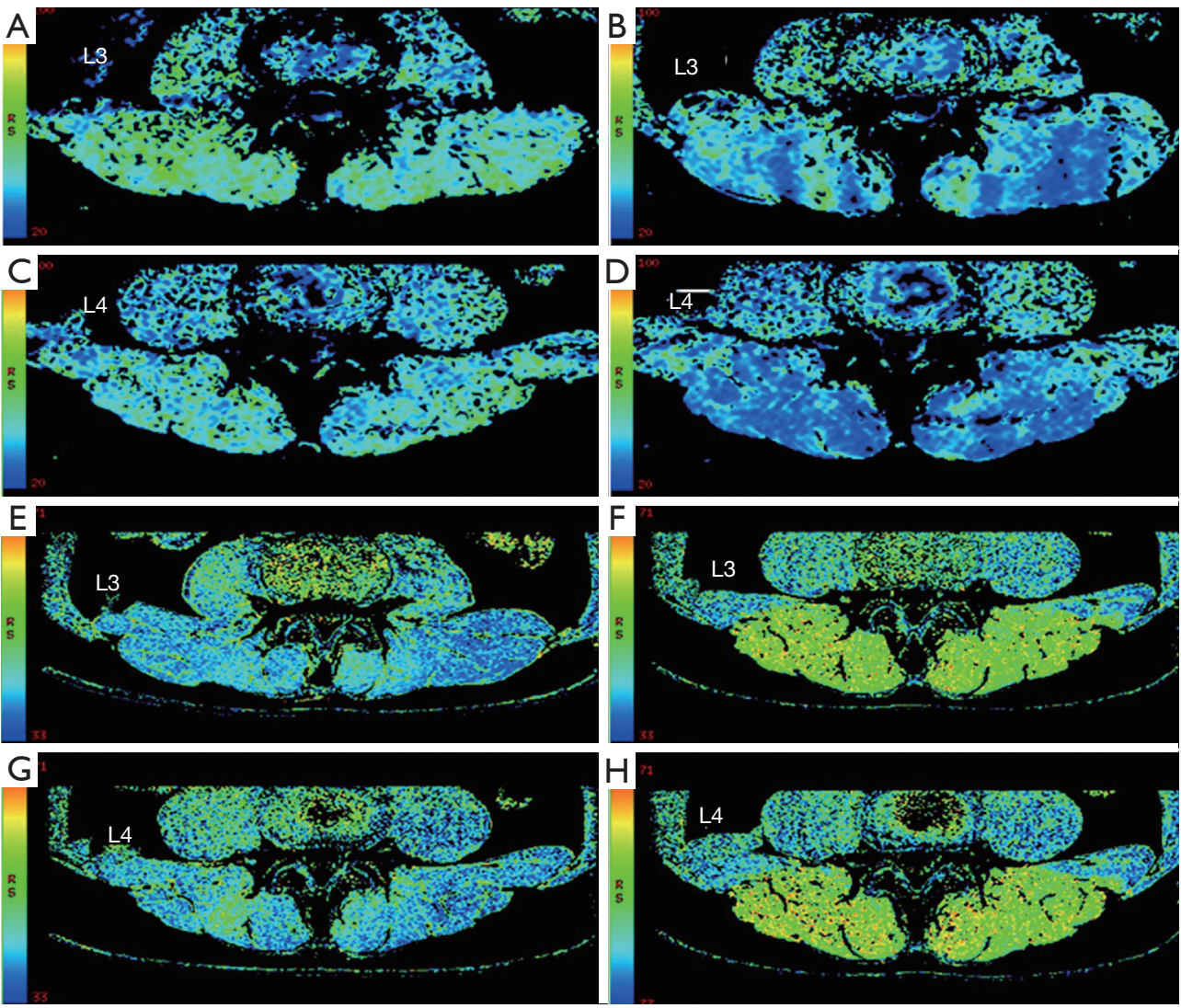

Figure 4 Color-coded R2* and T2-calculated maps. In R2* maps, blue represents areas of lower R2* value, and red represents areas of high R2* value. R2* maps obtained before exercise at L3 (A) and L4 (C); R2* maps obtained after exercise showing a marked decrease in the total R2* values of paraspinal muscles at L3 (B) and L4 (D), on both left and right sides, indicating diminished deoxyhemoglobin in paraspinal muscles. In the T2-calculated map, blue represents areas of short T2 values, and red represents areas of long T2 values. T2 maps obtained before (E) and after (F) exercise at L3; T2 maps obtained before (G) and after (H) exercise at the L4 level. The range of color bar of R2* maps is 20-100, and the range of color bar of T2 maps is 33-71.

muscles (multifidus, longissimus, and iliocostalis) before and after exercise. The CSA, R2*, and T2 between males and females, left and right sides, and L3 and L4 levels, were compared using the independent-samples $t$-test.

\section{Results}

\section{Analysis of CSA, R2*, and T2 exercise values}

The values of CSA, R2*, and T2 were obtained before and after exercise, as described earlier. Significant changes and differences in these parameters were observed across the iliocostalis, longissimus, and multifidus muscles before and after exercise sessions (Figure 3C,D,E,F, Figure 4, and Tables 3,4,5). The CSA of the lower-back muscles on the L3-
L4 levels significantly differed between the sessions. CSA values on the $\mathrm{L} 3$ level were in the following order: iliocostalis > longissimus > multifidus; for the L4 level: iliocostalis > multifidus $>$ longissimus. The CSA of the post-exercise session was higher than the pre-exercise session for all lowerback muscles (all $\mathrm{P}<0.001$; Table 3, Figure 3C,D,E,F).

The $\mathrm{R} 2 *$ values decreased for all lower-back muscles after exercise at both the L 3 and L4 levels (all $\mathrm{P}<0.001$; Table 4, Figure 4A,B,C,D, Table S1). However, these decreases were not significant. The T2 of the paraspinal muscles significantly increased after exercise; the order was multifidus $>$ longissimus $=$ iliocostalis. Similarly, the postexercise T2 values were significantly higher than those of the pre-exercise session (all $\mathrm{P}<0.001$; Table 5, Figure $4 E, F, G, H)$. 
Table 3 CSA of paraspinal muscles before and after exercise $\left(\mathrm{n}=50\right.$, mean \pm standard deviation, $\left.\mathrm{mm}^{2}\right)$

\begin{tabular}{lccccc}
\hline Spinal level & Session & lliocostalis & Longissimus & Multifidus & CSA (total) \\
\hline L3 & Pre-exercise & $830.39 \pm 247.00$ & $491.72 \pm 184.84$ & $263.04 \pm 67.98$ & $1,585.16 \pm 430.68$ \\
& Post-exercise & $876.11 \pm 268.67$ & $551.69 \pm 202.33$ & $302.26 \pm 76.77$ & $1,730.05 \pm 471.72$ \\
& P value & $0.000^{*}$ & $0.000^{*}$ & $0.000^{*}$ & $0.000^{*}$ \\
L4 & Pre-exercise & $847.51 \pm 240.79$ & $366.00 \pm 122.14$ & $409.07 \pm 114.68$ & $1,622.59 \pm 376.14$ \\
& Post-exercise & $906.90 \pm 273.4$ & $412.43 \pm 122.54$ & $461.23 \pm 137.24$ & $1,780.56 \pm 446.35$ \\
& P value & $0.000^{*}$ & $0.000^{*}$ & $0.000^{*}$ & $0.000^{*}$ \\
\hline
\end{tabular}

CSA, cross-sectional area. *All $\mathrm{P}<0.05$.

Table 4 R2* of paraspinal muscles before and after exercise ( $\mathrm{n}=50$, mean \pm standard deviation, $\mathrm{s}^{-1}$ )

\begin{tabular}{lcccc}
\hline Spinal level & Session & lliocostalis & Longissimus & Multifidus \\
\hline L3 & Pre-exercise & $50.57 \pm 11.91$ & $48.99 \pm 10.67$ & $48.50 \pm 11.38$ \\
& Post-exercise & $46.63 \pm 11.50$ & $44.04 \pm 10.49$ & $44.20 \pm 10.65$ \\
P value & $0.000^{*}$ & $0.000^{*}$ & $0.000^{*}$ \\
L4 & Pre-exercise & $46.49 \pm 7.95$ & $45.90 \pm 7.65$ & $47.32 \pm 8.69$ \\
& Post-exercise & $41.88 \pm 8.53$ & $39.52 \pm 7.92$ & $40.09 \pm 9.31$ \\
& P value & $0.000^{*}$ & $0.000^{*}$ & $0.000^{*}$ \\
\hline
\end{tabular}

${ }^{*}$ All $\mathrm{P}<0.05$.

Table 5 T2 value of paraspinal muscles before and after exercise ( $\mathrm{n}=50$, mean \pm standard deviation, ms)

\begin{tabular}{lcccc}
\hline Spinal level & Session & lliocostalis & Longissimus & Multifidus \\
\hline L3 & Pre-exercise & $47.67 \pm 5.68$ & $54.46 \pm 10.97$ & $70.16 \pm 38.8$ \\
& Post-exercise & $52.62 \pm 5.85$ & $61.05 \pm 10.68$ & $76.93 \pm 24.57$ \\
& P value & $0.000^{*}$ & $0.000^{*}$ & $0.000^{\star}$ \\
L4 & Pre-exercise & $47.31 \pm 6.72$ & $50.87 \pm 6.47$ & $55.43 \pm 16.89$ \\
& Post-exercise & $51.98 \pm 6.40$ & $57.1 \pm 5.98$ & $62.21 \pm 9.77$ \\
& P value & $0.000^{*}$ & $0.000^{*}$ & $0.000^{*}$ \\
\hline
\end{tabular}

${ }^{*}$ All $\mathrm{P}<0.05$.

\section{Analysis of CSA, R2*, and T2 regarding sex and anatomical locations}

Comparisons of the pre- and post-exercise CSA, R2*, and T2 forsex, L3-L4 levels, and left and right sides are shown in Figures 5,6,7. There were demonstrable differences in these parameters in the iliocostalis, longissimus, and multifidus muscles after exercise. In all investigated muscles, higher post-exercise CSA and T2 levels and lower postexercise R2* levels were observed in both male and female participants, L3 and L4 levels, and both left and right sides (all $\mathrm{P}<0.05$ ). Males displayed larger CSA and lower R2* values on all spinal levels both at rest and after exercise (all $\mathrm{P}<0.001$ ) (Figures 5,6). No significant differences in the T2 values were observed between males and females (Figure 7).

The differences in the $\Delta \mathrm{CSA}, \Delta \mathrm{R} 2^{*}$, and $\Delta \mathrm{T} 2$ values were evident in the iliocostalis muscles between males and females. Between pre- and post-exercise sessions, the multifidus CSA of the L4 level was larger than that of the 

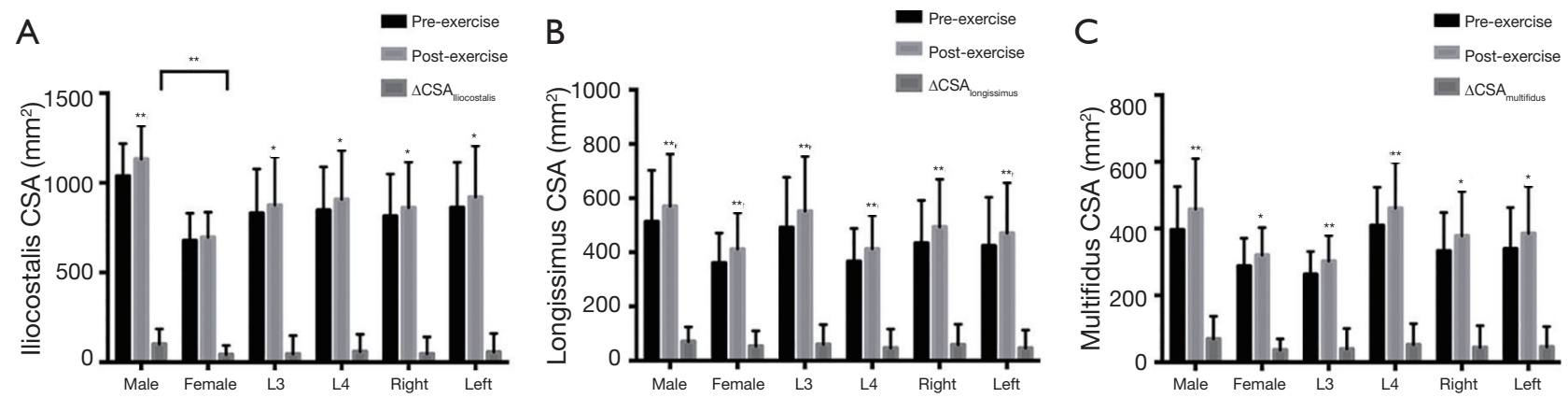

Figure 5 Comparison of CSA values of the three paraspinal muscles between pre- and post-exercise sessions with respect to sex, spinal level, and sides. (A) Iliocostalis; (B) longissimus; (C) multifidus. Data are reported as mean \pm standard error of mean; ${ }^{* *} \mathrm{P}<0.01$; ${ }^{*} \mathrm{P}<0.05$ (independent samples $t$-test).
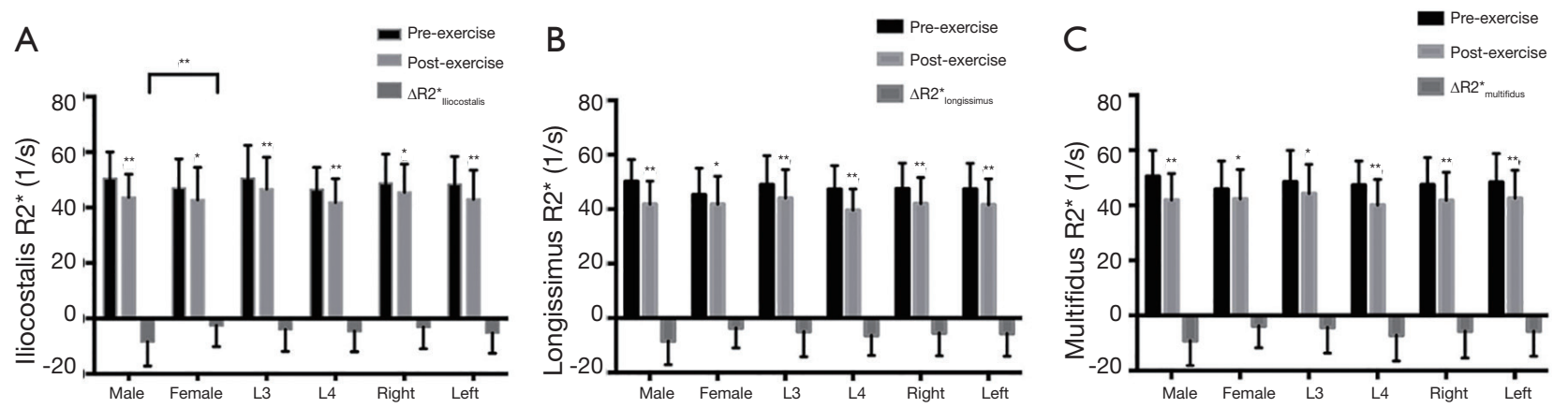

Figure 6 Comparison of R2* values of the three paraspinal muscles between pre- and post-exercise sessions with respect to sex, spinal level, and sides. (A) Iliocostalis; (B) longissimus; and (C) multifidus. Data are reported as mean \pm standard error of mean; ${ }^{* *} \mathrm{P}<0.01 ;{ }^{*} \mathrm{P}<0.05$ (independent samples $t$-test).
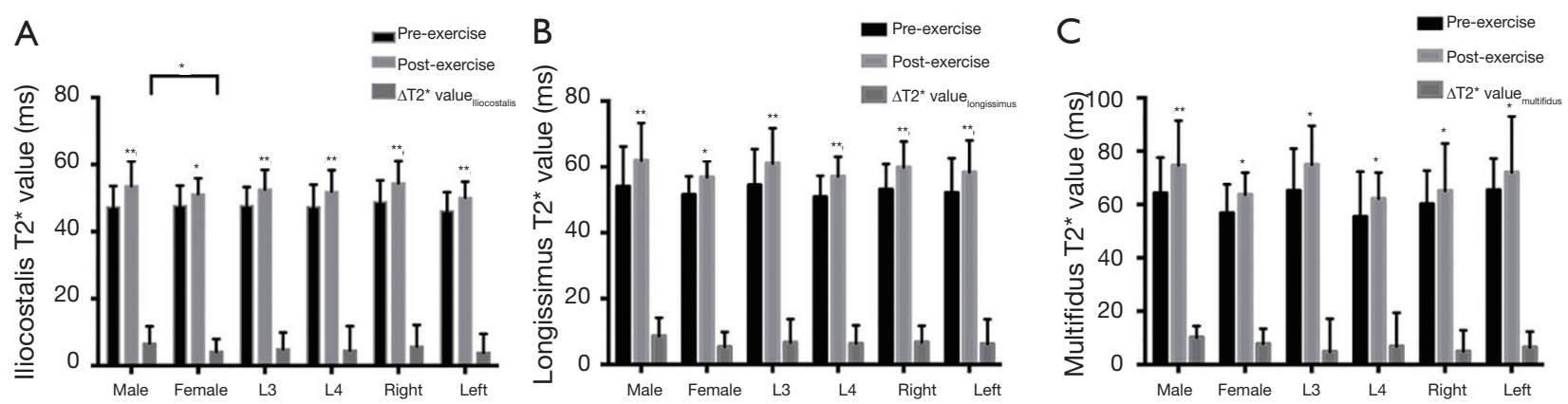

Figure 7 Relative changes in the T2 values of the paraspinal muscles before and after exercise with respect to sex, spinal level, and sides. (A) Iliocostalis; (B) longissimus; and (C) multifidus. Data are reported as mean \pm standard error of mean; ${ }^{* *} \mathrm{P}<0.01$; ${ }^{*} \mathrm{P}<0.05$ (independent samples $t$-test).

L3 level $(\mathrm{P}<0.05)$, and the longissimus CSA on the L3 level was larger than that of the $\mathrm{L} 4$ level $(\mathrm{P}<0.001)$. Regardless of sex or L3-L4 spinal levels, significant differences were observed in the CSA, R2*, and T2 between the left and right muscles following exercise $(\mathrm{P}<0.05)$. However, no significant differences in any of the measurements were noticed between the left and right muscles at either pre- or post-exercise (Figures 5,6,7). Comparisons of the pre- and 
post-exercise 1/R2* regarding sex, L3 and L4 levels, and left and right sides are shown in Figure S1.

\section{Discussion}

Our study examined exercise-induced changes in the lower back muscles. We found that parameters of muscle fMRI (BOLD and T2 mapping) are feasible alternatives to traditional CSA measurements in evaluating muscle function. Our technique may provide a useful imaging tool for the evaluation of treatment efficacy and recovery progression in patients with lower-back pain. In addition, upper body flexion and extension using a simple Roman chair can activate the lower back muscles, which can be detected by fMRI.

Both regular and acute exercise can increase muscle CSA (16). In our study, the acute exercise caused blood vessel dilation and increased blood perfusion. In addition, the accumulation of metabolites (such as lactic acid) can increase the osmotic pressure in myocytes, leading to higher water content (17). All these changes can increase CSA in the paraspinal muscles in our study.

All groups (male/female, L3/L4 levels, right/left sides) displayed higher CSA and T2 values and lower R2* values in all lower-back muscles after exercise. Notably, following lower back hyperextension exercises, there was a rapid decrease in BOLD signal intensity in all muscle groups. Similar decreases in the BOLD signal of calf muscles were observed in a plantar flexion study by Schewzow et al. (18). In addition, a study by Muller et al. performed in 6healthy men demonstrated that changes in the BOLD signal were closely related to the intensity of the dynamic plantar flexion exercise (19). Observed BOLD signal and T2 value changes may arise from a variety of factors, including dynamic changes in muscle microcirculatory blood perfusion, blood flow, oxygenation levels, and biological metabolism (20).

Laser Doppler flowmeter (LDF) is a relatively accurate method for evaluating muscle microcirculatory blood perfusion, but the application of the technique is confined to the skin and shallow muscle tissue (21). Moreover, the blood flow of partial and superficial tissues is not representative of the whole muscles of the lower back. The use of LDF is also invasive as it requires inserting the probe into the muscle $(19,20)$. In contrast, BOLD is primarily dependent on the deoxyhemoglobin content, a paramagnetic substance that allows BOLD to indirectly measure the oxygenation status and microcirculatory blood perfusion of tissue, through the detection of $\mathrm{R} 2 *$ changes
$(7,10)$. Compared to LDF, BOLD allows the assessment of biological metabolic changes in deep muscle tissue and allows accurate anatomical mapping when superimposed onto high-resolution anatomical MRI (22). It is an imaging technique widely used in studies of the brain, myocardial ischemia, liver cancer, and skeletal muscles $(7,12,14,23,24)$.

Activity-related changes in T2 mapping reflect increased T2 relaxation times of skeletal muscle (25). In addition, T2 mapping can be used as a quantitative metric for the evaluation of various muscle functions. For example, Hiepe et al. reported that an increase in $\mathrm{T} 2$ following spinal muscle movements were related to changes in muscle metabolism $(26,27)$, the number of capillaries, and the degree of fat infiltration in the muscle. The changes in $\mathrm{T} 2$ values after exercise observed in our study are consistent with these findings. Following skeletal muscle exercise, osmotic pressure in the muscle cells increases due to increased levels of muscle cell metabolites (for example, lactic acid), resulting in increased water content in the extracellular space.

Moreover, the increase in muscle $\mathrm{T} 2$ is related to the degree of muscle stimulation and contraction. Mayer showed that T2 values are higher in spinal muscle during increased exercise intensity (28). In this study, the increased $\mathrm{T} 2$ of the superior margin of L3 and L4 following exercise may be related to the presence of lactic acid and water following muscle activation. This may also explain the observed increase in muscle CSA which reflects the overall change in muscle morphology after exercise.

T2 maps are determined by the metabolism of water molecules in the muscles. Meanwhile, R2* maps are determined by deoxyhemoglobin in the blood, which reflects the changes in oxygen levels. However, T2 maps and $\mathrm{R} 2 *$ maps are not independent of each other. This is because exercise can alter water metabolism, blood perfusion, and deoxyhemoglobin.

Although changes in the BOLD and T2 mapping of muscle following exercise witnessed in this study provide insight into the changes in muscle activation, $\mathrm{R} 2^{*}$ and $\mathrm{T} 2$ values can also be affected by muscle microcirculatory blood perfusion, blood volume, extracellular water content, and other biological factors. Therefore, the specific effects of this range of factors regarding muscle activation warrant further investigation.

The present study is not without limitations. Firstly, the number of participants was limited-a factor that may impact the observations made. Secondly, BOLD and T2mapping cannot be accomplished simultaneously 
following paraspinal muscle exercise, meaning there was a time lag between measurements that may introduce some degree of inaccuracy. Thirdly, only young, healthy volunteers with an age range of 19-29 years were enrolled. Thus, our data may not extend to other age groups.

In conclusion, BOLD and T2 mapping provide useful functional information that makes these techniques appealing in evaluating the activation of paraspinal muscles. They can provide deeper insights into muscle physiology and complement the use of CSA.

\section{Acknowledgments}

None.

\section{Footnote}

Conflicts of Interest: The authors have no conflicts of interest to declare.

Ethical Statement: Our study was approved by the ethics committee of our hospital. All participants gave written informed consent before the study.

\section{References}

1. Mannion AF, Junge A, Taimela S. Active Therapy for Chronic Low Back Pain. Spine (Phila Pa 1976) 2001;26:920-9.

2. Storheim K, Holm I, Gunderson R, Brox JI, Bø K. The effect of comprehensive group training on cross-sectional area, density, and strength of paraspinal muscles in patients sick-listed for subacute low back pain. J Spinal Disord Tech 2003;16:271-9.

3. Keller A, Gunderson R, Reikerås O, Brox JI. Reliability of computed tomography measurements of paraspinal muscle cross-sectional area and density in patients with chronic low back pain. Spine (Phila Pa 1976) 2003;28:1455-60.

4. Motosuneya T, Asazuma T, Tsuji T, Watanabe H, Nakayama Y, Nemoto K. Postoperative change of the cross-sectional area of back musculature after 5 surgical procedures as assessed by magnetic resonance imaging. $\mathrm{J}$ Spinal Disord Tech 2006;19:318-22.

5. Parkkola R, Rytökoski U, Kormano M. Magnetic resonance imaging of the discs and trunk muscles in patients with chronic low back pain and healthy control subjects. Spine (Phila Pa 1976) 1993;18:830-6.

6. Hu ZJ, He J, Zhao FD, Fang XQ, Zhou LN, Fan SW.
An assessment of the intra- and inter-reliability of the lumbar paraspinal muscle parameters using CT scan and magnetic resonance imaging. Spine (Phila $\mathrm{Pa} 1976$ ) 2011;36:E868-74.

7. Ogawa S, Lee TM, Kay AR, Tank DW. Brain magnetic resonance imaging with contrast dependent on blood oxygenation. Proc Natl Acad Sci U S A 1990;87:9868-72.

8. Glover GH. Overview of Functional Magnetic Resonance Imaging. Neurosurg Clin N Am 2011;22:133-9.

9. Ledermann HP, Heidecker HG, Schulte AC, Thalhammer C, Aschwanden M, Jaeger KA, Scheffler K, Bilecen D. Calf muscles imaged at BOLD MR: correlation with TcPO2 and flowmetry measurements during ischemia and reactive hyperemia--initial experience. Radiology 2006;241:477-84.

10. Partovi S, Schulte AC, Jacobi B, Kenmoku T, Ochiai N, Obata T, Toyone T, Wada Y, Okubo T. Blood oxygenation level-dependent (BOLD) MRI of human skeletal muscle at 1.5 and 3 T. J Magn Reson Imaging 2012;35:1227-32.

11. Matsuki K, Watanabe A, Ochiai S, et al. Quantitative evaluation of fatty degeneration of the supraspinatus and infraspinatus muscles using T2 mapping. J Shoulder Elbow Surg 2014;23:636-41.

12. Ledermann HP, Schulte AC, Heidecker HG, Aschwanden M, Jäger KA, Scheffler K, Steinbrich W, Bilecen D. Blood oxygenation level-dependent magnetic resonance imaging of the skeletal muscle in patients with peripheral arterial occlusive disease. Circulation 2006;113:2929-35.

13. Glaser C. New techniques for cartilage imaging: $T 2$ relaxation time and diffusion-weighted MR imaging. Radiol Clin North Am 2005;43:641-53, vii.

14. Kim HK, Laor T, Horn PS, Racadio JM, Wong B, Dardzinski BJ. T2 mapping in Duchenne muscular dystrophy: distribution of disease activity and correlation with clinical assessments. Radiology 2010:255:899-908.

15. Manka R, Paetsch I, Schnackenburg B, Gebker R, Fleck $\mathrm{E}$, Jahnke C. BOLD cardiovascular magnetic resonance at 3.0 tesla in myocardial ischemia. J Cardiovasc Magn Reson 2010;12:54.

16. Wroblewski AP, Amati F, Smiley MA, Goodpaster B, Wright V. Chronic exercise preserves lean muscle mass in masters athletes. Phys Sportsmed 2011;39:172-8.

17. Mukaimoto T, Semba S, Inoue Y, Ohno M. Changes in transverse relaxation time of quadriceps femoris muscles after active recovery exercises with different intensities. J Sports Sci 2014; 32:766-75.

18. Schewzow K, Fiedler GB, Meyerspeer M, Goluch S, Laistler E, Wolzt M, Moser E, Schmid AI. Dynamic ASL and $\mathrm{T}^{*}{ }^{*}$-weighted $\mathrm{MRI}$ in exercising calf muscle at $7 \mathrm{~T}$ : A 
feasibility study. Magn Reson Med 2015;73:1190-5.

19. Muller MD, Li Z, Sica CT, Luck JC, Gao Z, Blaha CA, Cauffman AE, Ross AJ, Winkler NJ, Herr MD, Brandt K, Wang J, Gallagher DC, Karunanayaka P, Vesek J, Leuenberger UA, Yang QX, Sinoway LI.Muscle oxygenation during dynamic plantar flexion exercise: combining BOLD MRI with traditional physiological measurements. Physiol Rep 2016;4:e13004.

20. Hennig J, Scheffler K, Schreiber A. Time resolved observation of BOLD effect in muscle during isometric exercise. Proc Int Soc Magn Reson Med 1999;8:122.

21. Blackwood SJ, Dwyer RM, Bradley EA, Keske MA, Richards SM, Rattigan S. Determination of Skeletal Muscle Microvascular Flowmotion with ContrastEnhanced Ultrasound. Ultrasound Med Biol 2017;43:2013-23.

22. Kesavadas C, Thomas B. Clinical applications of functional MRI in epilepsy. Indian J Radiol Imaging 2008;18:210-7.

23. Stacy MR, Qiu M, Papademetris X, Caracciolo CM, Constable RT, Sinusas AJ. Application of BOLD Magnetic Resonance Imaging for Evaluating Regional Volumetric Foot Tissue Oxygenation: A Feasibility Study in Healthy

Cite this article as: Huang YL, Zhou JL, Jiang YM, Zhang ZG, Zhao W, Han D, He B. Assessment of lumbar paraspinal muscle activation using fMRI BOLD imaging and T2 mapping. Quant Imaging Med Surg 2020;10(1):106-115. doi: 10.21037/ qims.2019.10.20
Volunteers. Eur J Vasc Endovasc Surg 2016;51:743-9.

24. Bane O, Besa C, Wagner M, Oesingmann N, Zhu H, Fiel MI, Taouli B. Feasibility and reproducibility of BOLD and TOLD measurements in the liver with oxygen and carbogen gas challenge in healthy volunteers and patients with hepatocellular carcinoma. J Magn Reson Imaging 2016;43:866-76.

25. Patten C, Meyer RA, Fleckenstein JL. T2 mapping of muscle. Semin Musculoskelet Radiol 2003;7:297-305.

26. Hiepe P, Gussew A, Rzanny R, Kurz E, Anders C, Walther M, Scholle HC, Reichenbach JR. Age-related structural and functional changes of low back muscles. Exp Gerontol 2015;65:23-34.

27. Hiepe P, Gussew A, Rzanny R, Anders C, Walther M, Scholle HC, Reichenbach JR. Interrelations of muscle functional MRI, diffusion-weighted MRI and 31PMRS in exercised lower back muscles. NMR Biomed 2014;27:958-70.

28. Mayer JM, Graves JE, Clark BC, Formikell M, PloutzSnyder LL. The use of magnetic resonance imaging to evaluate lumbar muscle activity during trunk extension exercise at varying intensities. Spine 2005;30:2556-63. 


\section{Supplementary}
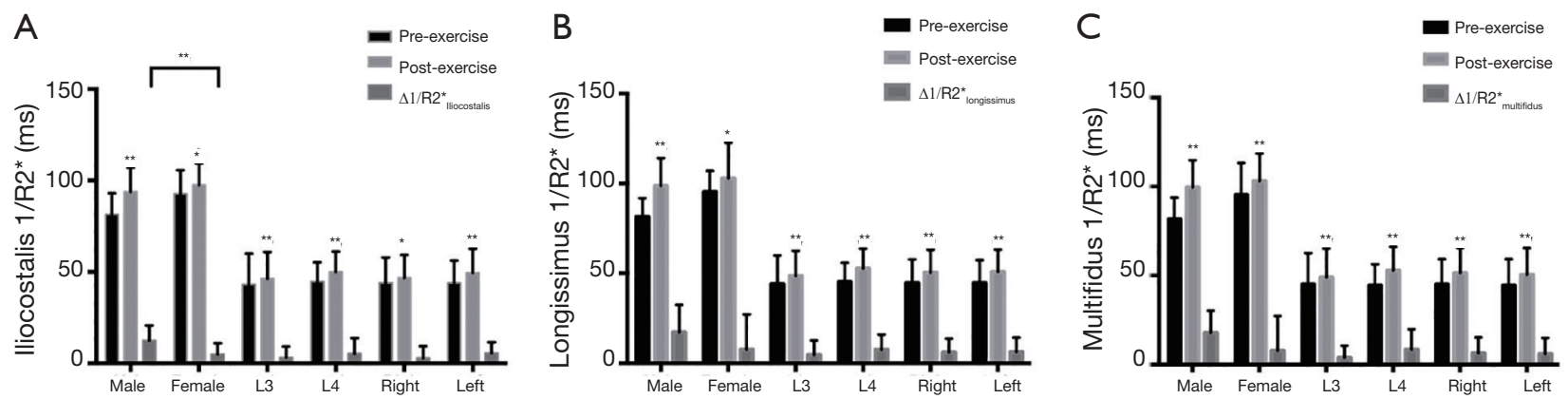

Figure S1 Comparison of 1/R2* values of the three paraspinal muscles between pre- and post-exercise sessions with respect to sex, spinal level, and sides. (A) Iliocostalis; (B) longissimus; and (C) multifidus. Data are reported as mean \pm standard error of mean; ${ }^{* *} \mathrm{P}<0.01$; ${ }^{*} \mathrm{P}<0.05$ (independent samples $t$-test).

Table S1 1/R2* of paraspinal muscles before and after exercise $(\mathrm{n}=50$, mean \pm standard deviation, $\mathrm{ms})$

\begin{tabular}{lcccc}
\hline Spinal level & Session & lliocostalis & Longissimus & Multifidus \\
\hline L3 & Pre-exercise & $43.01 \pm 16.87$ & $44.01 \pm 15.99$ & $45.07 \pm 17.54$ \\
& Post-exercise & $46.22 \pm 14.61$ & $48.56 \pm 13.92$ & $48.96 \pm 16.24$ \\
P value & $0.001^{*}$ & $0.000^{\star}$ & $0.000^{\star}$ \\
L4 & $44.69 \pm 10.48$ & $45.24 \pm 10.60$ & $44.38 \pm 11.93$ \\
& Pre-exercise & $49.93 \pm 11.10$ & $52.70 \pm 11.01$ & $52.87 \pm 13.22$ \\
& Post-exercise & $0.000^{*}$ & $0.000^{*}$ & $0.000^{*}$ \\
\hline
\end{tabular}

${ }^{*}$ All $\mathrm{P}<0.05$ 